\title{
Iot Based Smart Poultry Farming using Commodity Hardware and Software
}

\author{
Lata S. Handigolkar, M.L. Kavya and P.D. Veena
}

\begin{abstract}
This work aims to provide details on how to build an automated Environment Controlled Poultry Management System (ECPMS) using low cost commodity hardware and open source software. A comprehensive system was built using Raspberry Pi, used as a Linux embedded system board and Arduino-Uno board for interfacing with different sensors. The system has been thoroughly investigated for various physical parameters associated with effective poultry management which includes temperature, humidity, moisture content in the air and air quality. It was found that the system not only monitors these parameters, but also regulates these parameters effectively. The framework was observed to be very useful for farmers as they could easily access and control the system remotely using their handheld mobile devices. The system reduces human intervention, saves time, optimizes resource utilization and increases poultry production.
\end{abstract}

Keywords--- Linux, Embedded system, Raspberry Pi 3, Arduino, Sensors, Google Cloud Messenger (GCM), MOTION Streamer

\section{INTRODUCTION}

$I_{\mathrm{c}}^{\mathrm{N}}$ NDIA is considered as an agricultural wealthy country in terms of food and environmental resources. Nevertheless, such prosperity was gradually regressed directly contributing to a low agricultural productivity and farmer incomes. The farmers additionally lacked insights in agricultural marketing strategies and high quality production planning.

According to world's agricultural produce, chicken is the most favored produce, since it is a nutrient-rich food providing high protein, low fat and cholesterol, and lower energy than other kinds of poultries. Also, it is quite easy to look after and propagate its species. [2] For 5 years, the chicken production has been increasing on an average of $4.63 \%$ yearly because of standardized farming management and good manufacturing practices, leading to more chicken consumption and an increased export number of both domestic and international destinations. On the other hand, a lacking of labor in chicken production processes has affected fresh chicken export, which is found to be the principal problem. [3] Another significant obstacle can be wrong knowledge sharing and folk wisdom in chicken farming which effects efficiency.

Lata S. Handigolkar, Assistant Professor, Dept of ECE, GMIT, Davangere, India.

M.L. Kavya, UG scholar, Dept of ECE, GMIT, Davangere, India. P.D. Veena, UG scholar, Dept of ECE, GMIT, Davangere, India. DOI:10.9756/BIJSESC.8269
This study aims to set up a new model by using a modern technology applied to chicken farming known as a "Smart

Farm" or "Intelligent Farm", which is expected to clear up the IOT Based smart poultry farming using commadity hardware and software farming. Smart Farm could perceive any changed information derived from a semi-automatic microprocessor, alarming all notification to a connected personal computer. The farm monitoring could be conducted via application programs on smart phones for convenience use, time saving, and reduced labor dependency, cost.

\section{RELATED WORK}

This research has focused on the use of modern technology to help manage animal farming, which means farm management automation in various ways. Manakant Intarakamhaeng and et al [4] studied the model of farm management automation technology with RFID, Result; the adoption of RFID, or radio-frequency identification of objects and animals including 5 kinds of animal: cattle, buffalo, sheep, pigs and rabbits were successfully individually identified and recorded automatically.

Md Saifudaullah Bin Bahrudin and Rosni Abu Kassim [5] presented a fire alarm system in a real-time monitoring system that detected the presence of smoke in the air due to fire and captured images via a camera installed inside a room when a fire occurs. The embedded systems used to develop this fire alarm system were Raspberry Pi and Arduino Uno. The key feature of the system is the ability to remotely send an alert when a fire is detected. When the presence of smoke is detected, the system will display an image of the room state in a web page. The system will need the user confirmation to report the event to the Firefighter using a Short Message Service (SMS). The advantage of using this system is that it will reduce the possibility of false alert reported to the Firefighter. The camera will only capture an image, so this system will consume only a little storage and power.

Kumar and Hancke [6] presented an animal health monitoring system (AHMS) for monitoring the physiological parameters such as rumination, body temperature, and heart rate with surrounding temperature and humidity. The developed system could also analyze the stress level corresponding to thermal humidity index (THI). The IEEE802.15.4 and IEEE1451.2 standards based sensor module was also developed successfully. The zigbee device and

PIC18F455 microcontroller were used in the implementation of sensor module. The graphical user interface (GUI) is implemented in Lab VIEW 9.0 according to the IEEE1451.1 standard. The real time monitoring of physiological and behavioural parameters can be present on 
the GUI PC. The device is very helpful and a inexpensive health care of livestock. A prototype model was developed and tested with high accuracy results.

From the above. There are many new technologies that can be used in a more efficient management of farms. The authors study has focused on the importance of modern technology of Raspberry $\mathrm{Pi}$, Arduino and Smart Phone combined with chicken farming.

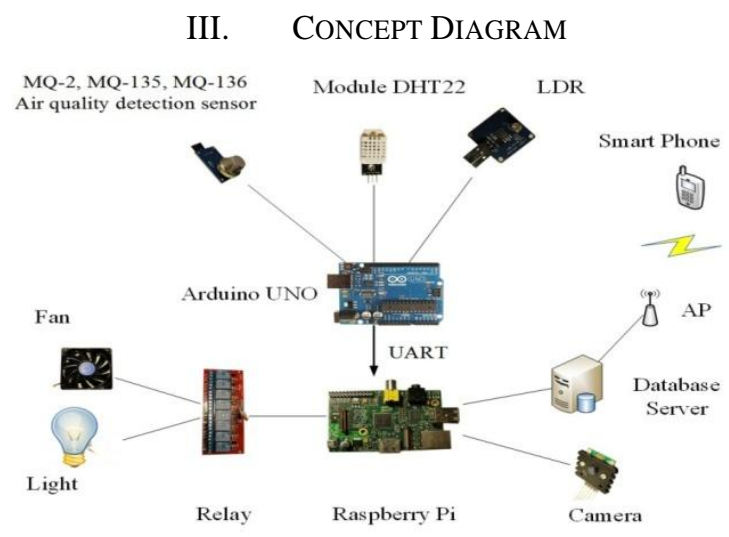

Figure 1: Full Block Diagram of the System

\section{SYSTEM OVERVIEW}

Shown in figure 1, components of the automatic farming system on Raspberry $\mathrm{Pi}$ Model-B and Arduino Uno are demonstrated. The system can notify using a real-time alarming system to smart phones reporting such as the current and daily highest/lowest temperature, humidity, and weather quality of the farm surroundings. Users can also control the filter fan switches and customize the notification system to the smart phone.

\section{Methodology}

A. Raspberry Pi

Raspberry Pi used in this work is Model 3 which is a revision of the original Raspberry $\mathrm{Pi}$. A Raspberry $\mathrm{Pi}$ is a credit-card sized computer originally designed for education, inspired by the 1981 BBC Micro. Raspberry pi 3 [4] is based on the Broadcom BCM2837 system on a chip (SoC) that includes an ARM Cortex-A53, 1.2GHz processor, Video Core 4 GPU, and having 1 GB LPDDR2, $900 \mathrm{MHz}$ RAM. It uses Micro SD card for boot media and relentless storage. The other features includes $700 \mathrm{MHz}$ clock speed, 4 individual USB 2.0 ports, 10/100 Base T Ethernet port, $2.4 \mathrm{GHz} 802.11 \mathrm{n}$ WIFI module, Low energy Bluetooth 4.1 module, HDMI audio and video output, has 40 GPIO pins used as both digital input or output operating at $3.3 \mathrm{~V}$.

\section{B. Arduino}

Arduino is an open-source prototyping platform based on easy-to-use hardware and software. In this work UNO variant of Arduino is used. Arduino UNO [5][6] is a microcontroller based developmental prototyping board, uses an ATMEGA328P controller chips having operating voltage of $5 \mathrm{~V}$, and has a clock speed of $10 \mathrm{MHz}$. Arduino Uno can be programmed with Arduino IDE, [7] as ATmega328 comes with preburned with a boot loader eliminates the need of using an external hardware programmer. Here each programme is known as sketch.

\section{Temperature Humidity Sensor Module}

Environmental conditions directly affect animal livelihood contributing to some chronic epidemics such as Bird Flu and Hand Foot and Mouth Disease. Therefore, DHT22 is use as a censor for measuring temperature (for both Fahrenheit and Celsius value) and humidity. The measurement unit will be demonstrated in a digital signal form.

\section{Gas Sensor Module}

The module works as a Air Quality Detection Gas Sensor, this is sensitive to gas dangerous to human, applied to measure NH3, NOx, Alchohol, Benzene, CO, and CO2. The module is also used for controlling weather conditions and air cleaners in buildings. The measurement unit is presented in a analog signal.

In this research, three censor forms were used, consisting of MQ-2, MQ-135, and MQ-136 because of its gas measurement differences.

\section{E. Photosensitive Sensor Module (LDR)}

A light sensor was used for measurement of light intensity especially for naked eye light, its unit is called Lux [8].

Light Dependent Resistor (LDR) is a light sensitive resistance changing electronic resistance when there is a light incidence, called Photo Resistor or Photo Conductor. The resistor was made from Semiconductor, Cadmium Sulfide (Cds) or Cadmium Selenide (CdSe). These two substances are semiconductors coated in a ceramic sheet as a base.

\section{F. Hardware Connection}

The Raspberry Pi and Arduino were connected via UART. The connection was a serial communication as Full Duplex since there was two-ways that data could be transmitted via pin TX and RX.

A direct connection between the Raspberry Pi and Arduino was prohibited, because of its electrical potential differences, which is 3.3 volts for the Raspberry $\mathrm{Pi}$ and 5 volts for the Arduino. Bi-directional Logic Level Converter should be used to separate them.

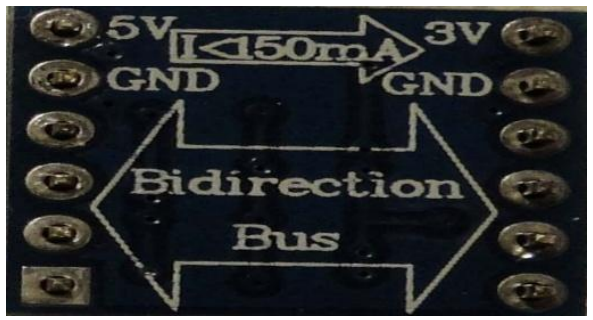

Figure 2: Bi-directional Logic Level Converter

\section{SYSTEM IMPLEMENTATION}

Building an ECPMS requires design, development and implementation on various hardware and software system. The system includes an Arduino UNO board continuously senses the data from the sensors connected to it, forwards it to 
Raspberry Pi over USB interface which analyses the data received, if physical parameters cross the threshold values, a data base entry is made and the same is notified to the user using GCM Service. User may respond to RPI server to take necessary action or may switch the system to auto mode.

The Raspberry Pi board is working as an co-coordinator node, installed with Rasbian wheezy which is a LINUX based Operating System (OS). This OS is installed on a SD card using SD formatter. SD formatter is used to format the card and win32diskimager to load the Operating system. After this, first boot configurations are made which includes root partition extension, head/headless mode booting, camera configuration, system update and upgrade.

In this architecture database server and web server are implemented on a single-board computer (RPI) using LAMP architecture [12], which reduces the complexity and cost of deployment. LAMP is an acronym used for Linux, Apache, My SQL and PHP is server configuration used to setup webapplication. It is a open source Web development platform that uses Linux as the operating system, at the heart of the LAMP stack is server software called Apache works as the Web server whose job is to process http requests, My SQL as the relational database management system and PHP as the object-oriented scripting language. These four Open-Source applications, commonly referred to as the LAMP stack, form a powerful and inexpensive alternative to proprietary website solutions. Nearly all hosting providers use a variation of the LAMP stack, whether it be shared hosting or a dedicated server. All the co-ordination functions are performed in Raspberry Pi implemented using python programming. Python receives data values from Arduino over serial port through USB interface. It then analyses to check whether the data is in permissible limits. If the data crosses the threshold limit, a data base entry is made and is notified to the user using GCM services.

In this work RPI needs to inform the user whenever sensor data crosses the threshold values in a reliable manner. Google Cloud Messaging (GCM) services [13] are used to push data from server to authorized users devices whenever abnormal condition is observed. GCM service handles all aspects of queuing of messages and delivery to client's applications running on target devices, and it is completely free. GCM architecture is illustrated in figure 2. GCM involves 2 main processes:

\section{Registration Process}

Android gadget sends client id, application id to GCM server to register the device.

On successful registration GCM server returns registration id Android gadget.

Now gadget will send the registration id to RPI server.

RPI server will store this registration id in its database for further usage.

\section{Notification Process}

When user is needed to be notified, RPI server pushes message to GCM server with device registration id.
GCM server then delivers this message to registered gadget using device registration id.

The web Applications are developed on the client side to provide users a convenient web interface to the system using Java frameworks and communicates with Raspberry Pi using a HTTP framework over GSM Internet. This application can fetch sensor values from Arduino and able to control light, fan and view video feed over his hand held device anywhere in the world. After notifying the user, RPI waits for a particular duration of time. If user instructs to perform the necessary action with the time, a particular GPIO pin is activated which is connected to a relay, drives an actuator (fan, light). If the user does not respond in specified time, the system will enter into auto mode.

Surveillance feature is implemented using a USB web camera and a command line service called motion used in Linux operating system used to monitor video signal from one or more cameras. We used a frame rate of $100 \mathrm{fps}$ and a resolution of $640 \times 480$. Output format used is .jpg where each frame overwrites the previous one hence saving in memory.

System is designed in such a way that coordinator node and sensors work independently of the web application. Legitimate user can access the sensor values from the data base by logging into Raspberry Pi. The web application can dynamically update the sensor values and plot the graphs without disturbing the sensors and actuators. This design architecture allow user to configure the system to a specific application with ease. Figure 3 shows the flow chart of overall system working

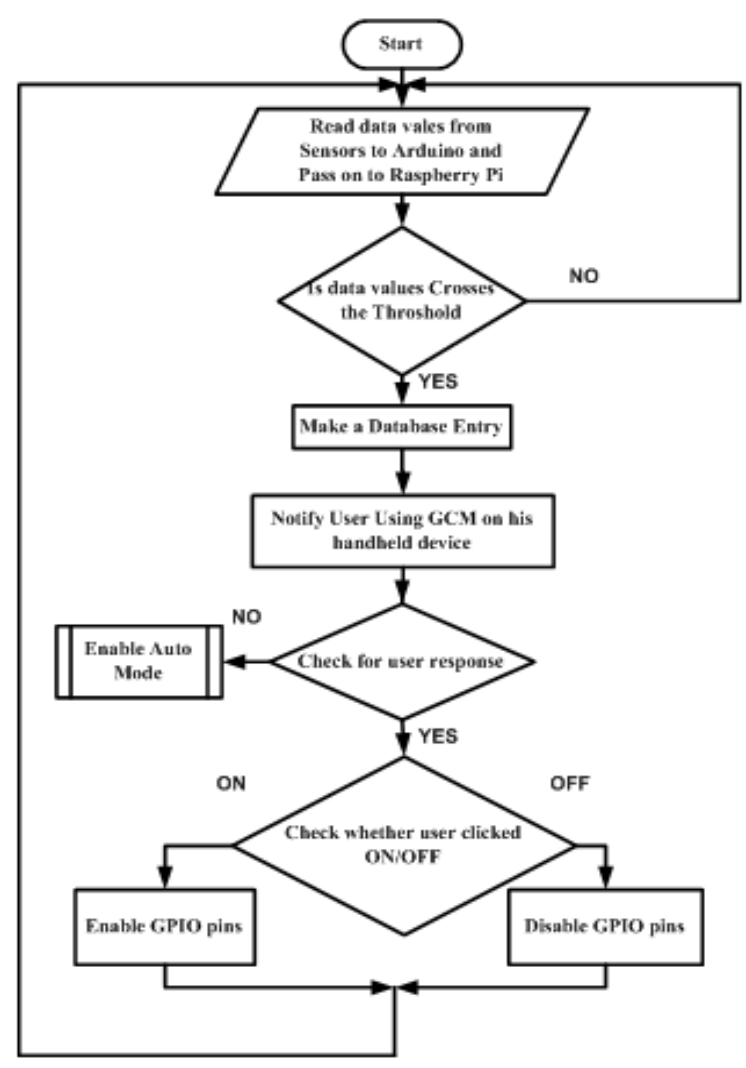

Figure 3: System Flow Chart 


\section{RESULTS AND DISCUSSION}

Deployment include placement of sensors, aggregator node, coordinator node and Actuators. Distance between two nodes is set to 10 meters. Figure 4 shows the snapshot of sensor setup with LCD facility for reading Sensor values. Figure 5 shows the connection between aggregator, coordinator node and actuator nodes.

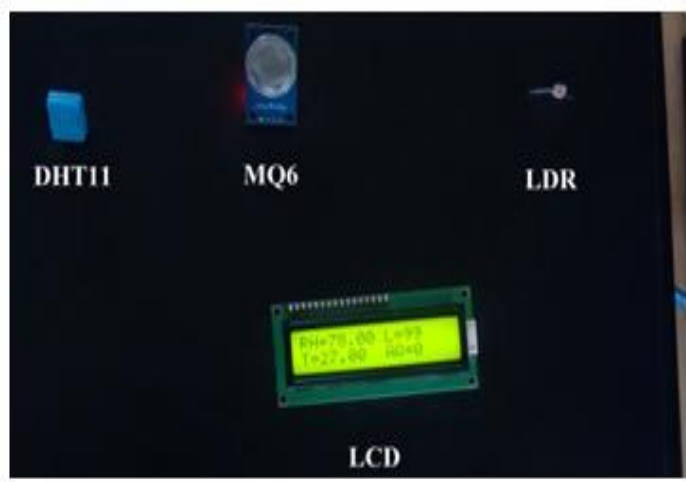

Figure 4: Sensor Setup Figure

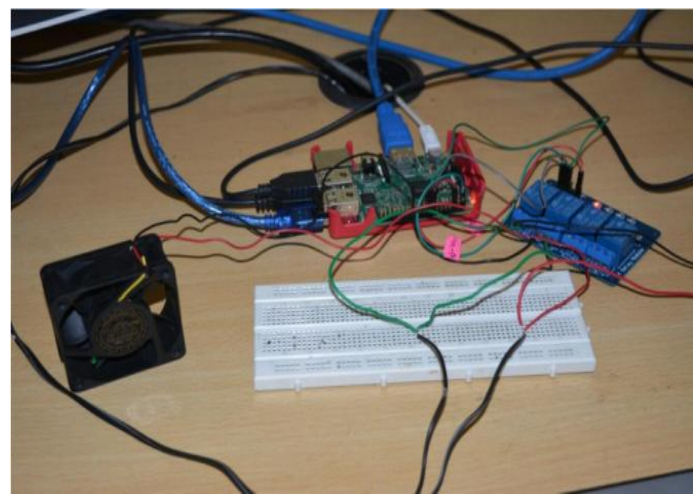

Figure 5: Connection between RPI and Actuator

Figure 6 shows the home screen of the user application where all the features namely mode (auto/manual), sensor stats, camera, graphs buttons are provided for rich user experience.

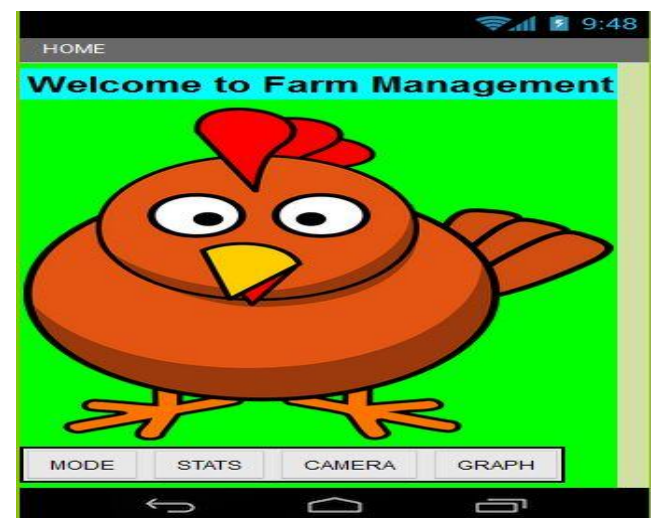

Figure 6: Home Screen of User App

Figure 7 show the graphical analysis of all the sensors implemented over a period of time.
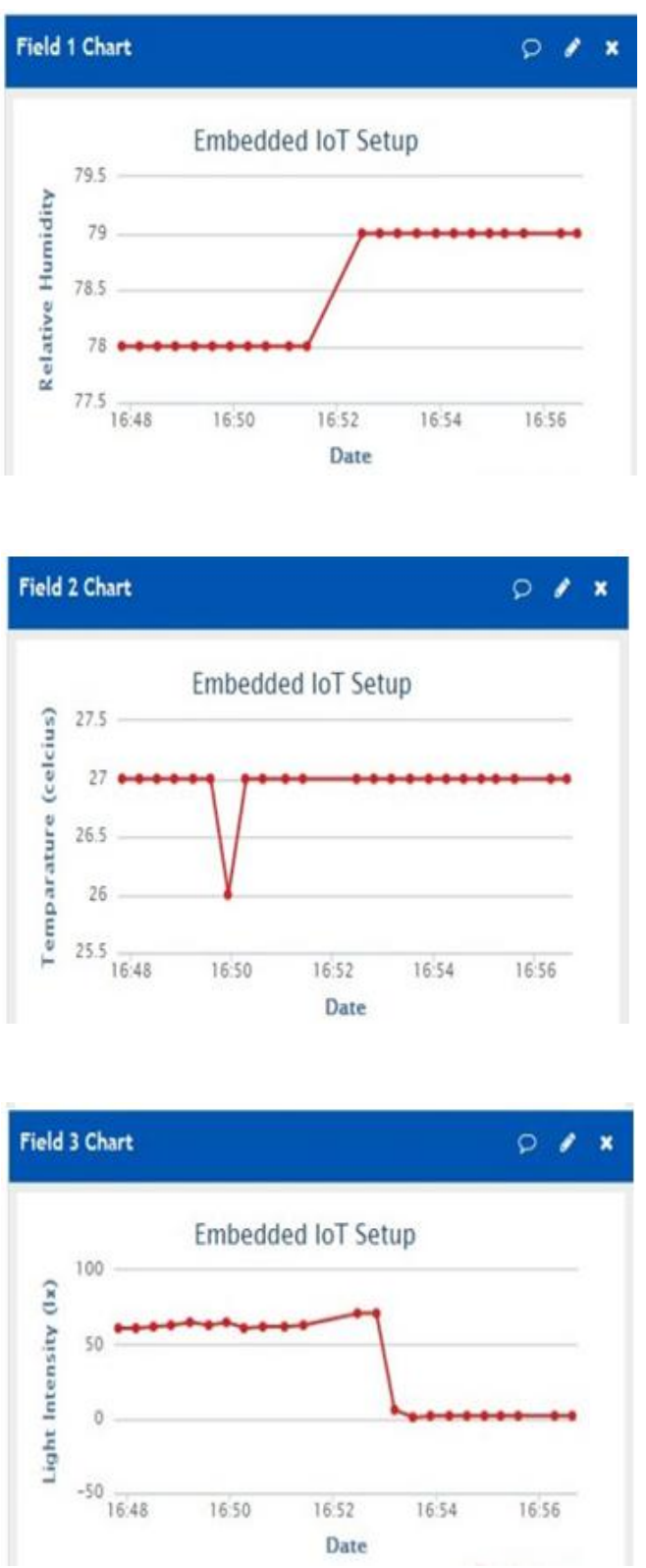

Figure 7: Sensor Values over Cloud for a Period of Time

Whenever a physical parameter crosses a predefined value, a notification is sent to user as shown in the figure 8

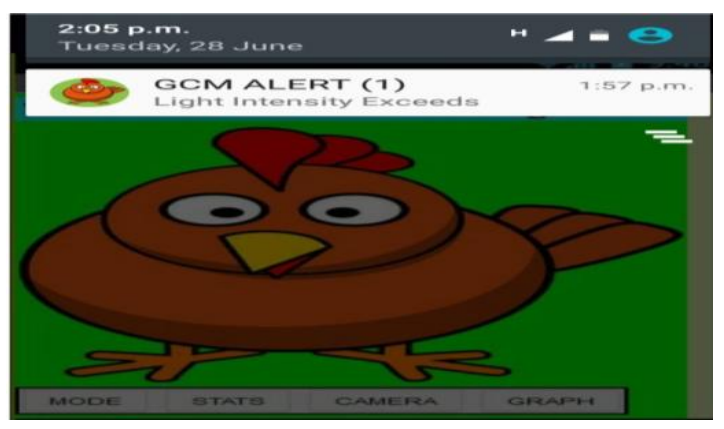

Figure 8:User Notification 
Table I shows the average measure of time elapsed in communication between coordinator node (RPI) and user device over different types of network.

Table I: Wi-Fi and Mobile Network Connection Message Delivery time (seconds)

\begin{tabular}{|l|l|l|l|}
\cline { 3 - 3 } \multicolumn{2}{c|}{} & \multicolumn{3}{c|}{$\begin{array}{c}\text { Connection Type (User } \\
\text { Device) }\end{array}$} \\
\cline { 3 - 4 } \multicolumn{2}{c|}{} & \multicolumn{1}{|c|}{ Wi-Fi } & \multicolumn{1}{c|}{ Cellular Data } \\
\hline $\begin{array}{l}\text { Connection Type } \\
\text { (RPI) }\end{array}$ & Wi-Fi & 4 & 13 \\
\cline { 2 - 4 } & $\begin{array}{l}\text { Cellular } \\
\text { Data }\end{array}$ & 11 & 16 \\
\hline
\end{tabular}

\section{CONCLUSION AND FUTURE WORK}

This Paper demonstrates an IoT system setup comprising Sensors, Arduino UNO, Raspberry Pi and Actuators. The system has lots of beneficiary features includes compact system, ease of deployment, maintenance, customization results in saving cost and human efforts. Integration of database server, web server and services on a single board computer (RPI) is one major advantage which reduces complexity of integration, deployment and management.

As a part of future work, communication between sensor module and coordinator can be made wireless by utilizing Bluetooth, xbee or wifi modules based on the requirement. The system architecture presented can be easily used in various automation designs with little modification to the need. As RPI has limited storage, cloud data storage can be used. The web interface can be developed to show the location of the sensor geographically and interface can be made better for rich user experience.

\section{REFERENCES}

[1] T.K. Hamrit, J.S. Durrence and G. Vellidis, "Precision Farming Practices", IEEE Industry Applications Magzine, Vol. 12, No. 2, Pp. 34 $-42,2009$.

[2] F.H. Fahmy, H.M. Farghally, N.M. Ahmed and A.A. Nafeh, "Modeling and Simulation of Evaporative Cooling System in Controlled Environment Greenhouse", Smart Grid and Renewable Energy, Pp. 6771, 2012.

[3] S. Don Kim and S. Eun Lee, "Little core Based system on Chip Platform for Tnternet of thing", International Journal of Electrical and Computer Engineering (IJECE), Vol. 5. No. 4, Pp. 695-700, 2015.

[4] "Raspberry pi 3 Specs, benchmarks and more", Mar. 1, 2016. [Online]. https://www.raspberrypi.org/magpi/raspberry-pi-3-specs-benchmarks/

[5] "Arduino UNO and Genuino UNO Documentation", https://www.arduino.cc/en/Main/ArduinoBoardUno

[6] V. Raja gogineni, K. Matcha and K. Raghava Rao, "Real Time Domestic Power Consumption Monitoring Using Wireless Sensor Networks", International Journal of Electrical and Computer Engineering (IJECE), Vol. 5. No. 4, Pp. 685-694, 2015.

[7] M. Banzi, "Getting Started with Arduino", $2^{\text {nd }}$ edition, O'Reilly Publication, September, 2011.

[8] "DHT11/21/22 etc Temperature and Humidity Sensors", [Online]: http://www.ladyada.net/learn/sensors/dht.html

[9] Gas Leakage detector MQ-6, https://www.sparkfun.com/products/9

[10] Modular Photoelectric Relays, http://www.pepperlfuchs.us/usa/en/16511.htm

[11] Light Dependent Resistors Working Principle, http://www.electrical4u.com/light-dependent-resistor-ldr-workingprinciple-of-ldr/

[12] $\mathrm{RPi}$ a simple LAMP, installation,http://elinux.org/RPi_A_Simple_Wheezy_LAMP_install.

[13] Y. Selim Yilmaz, B. Ismail Aydin and M. Demirbas, "Google cloud messaging (GCM): An evaluation", IEEE Conference Publication 2014 IEEE Global Communications Conference, Pp. 2807 - 2812, 2014. 